\title{
Investigations on Merging Breeds in Genetic Conservation Schemes
}

\author{
J. Bennewitz, ${ }^{*}{ }^{1}$ H. Simianer, $\ddagger$ and T. H. E. Meuwissen ${ }^{*}$ \\ *Department of Animal and Aquacultural Sciences, Norwegian University of Life Sciences, Box 1432, Ås, Norway \\ †nstitute of Animal Breeding and Husbandry, Christian-Albrechts-University of Kiel, 24098 Kiel, Germany \\ †Institute of Animal Breeding and Genetics, Georg-August-University Göttingen, 37075 Göttingen, Germany
}

\begin{abstract}
Genetic diversity within livestock species is threatened by extinction of breeds and by genetic drift; the need to conserve genetic diversity by conservation schemes is widely accepted. To maintain the betweenbreed diversity, breeds are usually kept separately in live conservation schemes. However, in some cases it might be very difficult or even impossible to conserve a highly endangered breed in a closed population. If this breed is important for diversity, it might be beneficial to merge it with one or more breeds to conserve a part of the diversity that is contributed by this breed. The present study introduces a general framework that may enable one to decide when it is beneficial to form a synthetic breed that includes highly endangered breeds to maximize conserved diversity and when to keep the breeds separate. Expected future diversities were estimated using a kinship-based diversity measure together with extinction probabilities of the breeds. Using a small hypothetical data set, the pattern of diversity and its 2 components, within-breed and between-breed diversity, were analyzed in detail when forming a synthetic breed. The suggested approach was applied to a data set of 13 central European red and yellow cattle breeds. The results suggested forming a synthetic breed by combining a nonendangered breed with 1 of the 2 highly endangered breeds, which would result in a slight increase in conserved diversity.
\end{abstract}

Key words: genetic diversity, conservation scheme, diversity measure, synthetic breed

\section{INTRODUCTION}

The genetic diversity within a livestock species can be divided into the diversity that can be found within and between breeds. The within-breed diversity is due to the different deviations from the respective breed mean, and the between-breed diversity arises from dif-

Received December 6, 2007.

Accepted February 27, 2008.

${ }^{1}$ Corresponding author: jorn.bennewitz@umb.no ferent breed means (Woolliams and Toro, 2007). It is often argued that the between-breed diversity, in particular, of farm animal species is threatened by extinction of breeds. For example, there are around 7,600 breeds from 35 domestic mammals and bird species. Around one-third of them are classified as endangered, at risk, or extinct (Scherf et al., 2006). Moreover, the within-breed diversity does not remain constant over time but declines with increasing inbreeding of the breed (Falconer and MacKay, 1996) and, of course, because of extinction.

The need to conserve genetic diversity is generally accepted (e.g., Oldenbroek, 2007). One major reason is that an abundant resource of genetic diversity is a prerequisite of coping with putative future changes in livestock farming conditions; for example, changes in production conditions or in market requirements. Because of limited resources it is not possible to conserve all endangered breeds. Therefore, an efficient conservation scheme should include only those breeds that contribute to its objectives (Simianer, 2005a), and the breeds included should be managed to minimize loss of within-breed diversity (Meuwissen, 2007). A very simple live conservation strategy is the establishment of a synthetic breed by interbreeding endangered breeds. The extinction probability of this synthetic breed would be low given that it is of sufficient size. Further, the inbreeding would be low and thus diversity within this breed high. However, such a strategy would destroy between-breed diversity and it is therefore generally recommended to keep the breeds separate in closed populations in conservation schemes (Meuwissen, 2007).

There are situations when it might be beneficial to merge endangered breeds in live conservation schemes. For example, consider 2 breeds $\mathrm{A}$ and $\mathrm{B}$, both with a high probability of extinction so that it is not desirable or even impossible to maintain them in a closed breeding population over the next decades even with the aid of conservation efforts and, therefore, these breeds would probably not contribute to diversity in the future. Now assume that both breeds would be merged resulting in the synthetic breed $\mathrm{AB}$, which might have a 
lower extinction probability compared with $\mathrm{A}$ and $\mathrm{B}$ and therefore, may have a reasonable chance of survival. In this case, the between-breed diversity of $\mathrm{A}$ and $\mathrm{B}$ will be lost, but because $\mathrm{AB}$ might survive, it will contribute to the future diversity, especially to the future withinbreed diversity. Hence, in terms of conserved diversity, it might be better to form $\mathrm{AB}$ than keeping $\mathrm{A}$ and $\mathrm{B}$ separate.

The objective of the present study was to introduce an approach that may enable breeders to decide when it is beneficial to form a synthetic breed that includes highly endangered breeds to maximize conserved diversity in live conservation schemes and when to keep the breeds separate. In particular, using a small hypothetical data set, the pattern of the total diversity and its 2 components, within-breed and between-breed diversity, was analyzed when forming a synthetic breed. The suggested approach was applied to a data set of 13 central European red and yellow cattle breeds.

\section{MATERIALS AND METHODS}

\section{General Diversity Concept}

Let $D_{0}$ be the present diversity that can be found in a set of $n$ breeds that are probably endangered, but still alive at present. $D_{0}$ is a weighted combination of the between-breed diversity and the within-breed diversities. The weights are determined by the diversity measure that is used. Further, let $E\left(D_{t}\right)$ be the expected diversity within this set at a future point in time $t$; for example, $t=25$ or 50 years into the future. Usually $D_{t}$ is different from $D_{0}$ because of extinction of breeds and genetic drift operating within the breeds. The extinction probability $z_{i}$ is the probability that breed $i$ will go extinct within $t$ yr. The amount of genetic drift operating in a breed within $t$ yr is a function of the effective population size $\left(N_{e}\right)$ and the generation interval (Falconer and MacKay, 1996). Let $m d_{i}$ be the marginal diversity of breed $i$, which is defined as the change of $E\left(D_{t}\right)$ when the extinction probability of the breed is lowered by 1 unit by a conservation effort. For $n$ being not too large, $E\left(D_{t}\right)$ and $m d$ can be estimated using the following approach (Simianer et al., 2003), ignoring the effect of genetic drift for the moment. Let $\mathbf{k}$ be an indicator vector of size $n$. Each element $k_{i}$ in $\mathbf{k}$ is allocated for one breed and obtains a zero if the breed is extinct at $t$ and a 1 otherwise. At $t$ there are $2^{n}$ different combinations of $k_{i}$ within possible $\mathbf{k}$; thus, $2^{n}$ different vectors might exist, each with a probability $P(\boldsymbol{k})$. For a certain vector $\boldsymbol{k}_{j}$ the probability is

$$
P\left(\mathbf{k}_{j}\right)=\prod_{i=1}^{n}\left(k_{i}+(-1)^{k_{i}} z_{i}\right)
$$

Let $D_{0}\left(\boldsymbol{k}_{j}\right)$ be the diversity of the set of breeds present in $\boldsymbol{k}$. Using this, the expectation of $D_{t}$ is

$$
E\left(D_{t}\right)=\sum_{j=1}^{2^{n}} P\left(\mathbf{k}_{j}\right) D_{0}\left(\mathbf{k}_{j}\right) .
$$

The marginal diversities are obtained by taking the partial derivatives of $E\left(D_{t}\right)$ with respect to $z$. For computational details see Simianer et al. (2003). Marginal diversities reflect the importance of the breeds for the expected future diversity. The idea of estimating marginal diversities and expected future diversity was proposed by Weitzman (1993).

Using this concept, each diversity measure, which is a nonnegative number and guarantees that $D_{0}$ does not increase if a breed is removed from a set, can be applied. In this study the maximum-variance-total (MVT) diversity measure (Bennewitz and Meuwissen, 2005a), which measures the expected total genetic variance of a hypothetical quantitative trait, was used. This measure was chosen because it allows a high degree of generality of the results obtained (see discussion section). It is based on average between and within breed kinships stored in a kinship matrix $\mathbf{M}$ of dimension $n$ by $n$. The method forms a core set in which the total genetic variance of a hypothetical quantitative trait that can be found within and between breeds is maximized. The relative contributions of the breeds to this core set are stored in the vector $\mathbf{c}_{\mathbf{m v t}}$ of dimension $n$ and can be estimated as

$$
\mathbf{c}_{\mathbf{m v t}}=\frac{1}{4}\left(\mathbf{M}^{-1} \mathbf{F}-\frac{\mathbf{1}_{\mathbf{n}}^{\prime} \mathbf{M}^{-1} \mathbf{F}-4}{\mathbf{1}_{\mathbf{n}}^{\prime} \mathbf{M}^{-1} \mathbf{1}_{\mathbf{n}}} \times \mathbf{M}^{-1} \mathbf{1}_{\mathbf{n}}\right),
$$

where $\mathbf{F}$ is a vector of dimension $n$ that contains the within-breed kinship; that is, $\mathbf{F}=\operatorname{diag}(\mathbf{M})$, and $\mathbf{1}_{\mathbf{n}}$ a vector of dimension $n$ containing $1 \mathrm{~s}$. The MVT diversity of the core set (subsequently denoted as $D$ ) is then calculated as

$$
D=1+\mathbf{c}_{\mathbf{m v t}}^{\prime} \mathbf{F}-2 \mathbf{c}_{\mathbf{m v t}}^{\prime} \mathbf{M} \mathbf{c}_{\mathbf{m v t}}
$$

The relative breed contributions reflect the importance of the breeds for the actual MVT diversity, $D$.

The expected future MVT diversity [subsequently denoted as $E\left(D_{t}\right)$ ] is estimated using equations [1] to [4], but replacing $\mathbf{M}$ and $\mathbf{F}$ by the expected kinship matrix and expected within-breed kinship vector at time $t, \mathbf{M}_{\mathrm{t}}$, and $\mathbf{F}_{\mathrm{t}}$, respectively. This accounts for the loss of withinbreed diversity due to drift operating in the breeds over the time horizon $t$, in addition to the loss due to extinction outlined above. Vector $\mathbf{F}_{t}$ can be modeled as a function of the rate of increase of within breed kinship due 
to genetic drift (stored in vector $\Delta \mathbf{F}$ ) and the number of generations $T$ (i.e., $t$ divided by the generation interval) as

$$
\mathbf{F}_{\mathbf{t}}=\mathbf{1}_{\mathbf{n}}-\left(\mathbf{1}_{\mathbf{n}}-\Delta \mathbf{F}\right)^{T} \bigcirc\left(\mathbf{1}_{\mathbf{n}}-\mathbf{F}\right),
$$

where $\bigcirc$ denotes for the Hadamard product. It was shown that the between-breed kinship (i.e., the off diagonals in $\mathbf{M}$ ) remain constant over time (Eding and Meuwissen, 2001). Hence, $\mathbf{M}_{\mathbf{t}}$ can be obtained by inserting the elements of $\mathbf{F}_{\mathbf{t}}$ into the corresponding diagonal elements of $\mathbf{M} . \Delta \mathbf{F}$ can be obtained as 1 divided by 2 times $N_{e}$ for each breed (Falconer and MacKay, 1996).

According to the redistribution of the variance of a quantitative trait in subdivided populations undergoing inbreeding (Falconer and MacKay, 1996, page 265), the MVT diversity can be partitioned into MVT between-breed diversity $\left(D_{b}\right)$ and MVT within-breed diversity $\left(D_{w}\right)$ as follows

$$
D_{b}=2\left(\mathbf{c}_{\mathbf{m v t}}^{\prime} \mathbf{F}-\mathbf{c}_{\mathbf{m v t}}^{\prime} \mathbf{M} \mathbf{c}_{\mathbf{m v t}}\right),
$$

and

$$
D_{w}=1-\mathbf{c}_{\mathbf{m v t}}^{\prime} \mathbf{F} .
$$

Hence, $D=D_{b}+D_{w}=2\left(\mathbf{c}_{\mathbf{m v t}}^{\prime} \mathbf{F}-\mathbf{c}_{\mathbf{m v t}}^{\prime} \mathbf{M} \mathbf{c}_{\mathbf{m v t}}\right)+(1-$ $\mathbf{c}_{\mathbf{m v t}}^{\prime} \mathbf{F}$ ), which is equivalent to equation [4]. The expected $D_{b}$ and expected $D_{w}$ at time $t, E\left(D_{b, t}\right)$ and $E\left(D_{w, t}\right)$, respectively, can be calculated using equations [1] to [6].

\section{Forming a Synthetic Breed}

It is assumed that a set of $m$ breeds, which is called $r$, will be combined into a synthetic breed called $s$. This is modeled by forming a kinship matrix $\mathbf{M}^{*}$ of size $n-$ $m+1$ by $n-m+1$. $\mathbf{M}^{*}$ is similar to $\mathbf{M}$ except that the kinships of the $m$ breeds are replaced through the kinships of the synthetic breed $s$. The average within breed kinship of the synthetic breed is obtained by

$$
\mathbf{M}_{\mathbf{s}, \mathbf{s}}^{*}=\mathbf{c}_{\mathbf{r}}^{\prime} \mathbf{M}_{\mathbf{r}, \mathbf{r}} \mathbf{c}_{\mathbf{r}}
$$

where matrix $\mathbf{M}_{\mathbf{r}, \mathbf{r}}$ contains the average kinships between and within the breed set $r$ and is the subsection of $\mathbf{M}$ pertaining to set $r$. Vector $\mathbf{c}_{\mathbf{r}}$ contains the contributions of each single breed to the synthetic breed. The elements in $\mathbf{c}_{\mathbf{r}}$ are nonnegative and their sum is equal to one. Similarly, the kinship between the synthetic breed and any other breed $x$ in the set is

$$
\mathbf{M}_{\mathbf{s}, \mathbf{x}}^{*}=\mathbf{c}_{\mathbf{r}}^{\prime} \mathbf{M}_{\mathbf{r}, \mathbf{x}}
$$

with $\mathbf{M}_{\mathbf{r}, \mathbf{x}}$ being the part of $\mathbf{M}$ pertaining to breeds $r$ and $x . D$ and $E\left(D_{t}\right)$ are then estimated using equations [1-6], but replacing $\mathbf{M}$ by $\mathbf{M}^{*}$. The modeling of the extinction probability of a synthetic breed will be shown below. The optimal values for $\mathbf{c}_{\mathbf{r}}$ are those that maximize $E\left(D_{t}\right)$ and these can be found iteratively.

As outlined in the introduction section, a combination of breeds in conservation schemes is an interesting option only in those situations where at least one breed is close to extinction and thus it might not be possible or meaningful to maintain it separately in a conservation scheme (e.g., due to too low population size). The following procedure for finding putative partners for endangered breeds is suggested.

Step 1: Select at random a set of $m$ breeds that includes at least one endangered breed and not more than one not-endangered breed.

Step 2: Form a synthetic breed by combining the $m$ breeds and estimate the expected diversity in this set $\left[E\left(D_{t}^{\prime}\right)\right]$. Calculate and store $\Delta E\left(D_{t}\right)=E\left(D_{t}^{\prime}\right)-E\left(D_{t}\right)$. Separate the $m$ combined breeds again.

Step 3: If any $\Delta E\left(D_{t}\right)>0$, combine those $m$ breeds that resulted in the largest $\Delta E\left(D_{t}\right)$ and update $E\left(D_{t}\right)=$ $E\left(D_{t}^{\prime}\right)$.

These 3 steps are repeated until no further improvements of $E\left(D_{t}\right)$ are found.

\section{Application to Simulated and Real Data Sets}

To illustrate how diversity behaves when breeds are combined into a synthetic breed, the methods were applied to a simulated data set. A base breed (breed 1) of effective size $N=50$ individuals with equal sex ratio was bred over 50 generations. Each subsequent generation was obtained by randomly sampling parents from the previous generation. Breed $2(N=50$ individuals $)$ was generated by sampling randomly parents from generation 20 of breed 1 and was bred for the next 30 generations separately. Similarly, breeds 3 and 4 of size $N=20$ and $N=18$, respectively, were generated by randomly sampling parents from generation 30 of breed 1 and were bred for the next 20 generations separately. The pedigree was used to set up the kinship matrix $\mathbf{M}$ using path analysis (Falconer and MacKay, 1996), see Table 1. The extinction probabilities were modeled as $z=\gamma$ $\times \frac{1}{N}$ with $\gamma=15$ (Table 1 ). The extinction probability of a synthetic breed $\left(z_{s}\right)$ was obtained as $z_{s}=\gamma \times \frac{1}{m}$. $\sum_{i=1} N_{i}$

The factor $\gamma$ was chosen arbitrarily so that extinction probability varies within a reasonable range. The future time horizon was $t=50 \mathrm{yr}$ and the generation 
Table 1. Size $(N)$, extinction probability $(z)$, average kinships, relative breed contribution to the maximumvariance-total (MVT) core set $\left(c_{m v t}\right)$, and marginal diversity $(m d)$ of the breeds from the hypothetical data set

\begin{tabular}{lcccccccc}
\hline & & \multicolumn{5}{c}{ Average kinship with breed } & & \\
\cline { 3 - 8 } Breed & $N$ & $z$ & 1 & 2 & 3 & 4 & $c$ & $m d^{1}$ \\
\hline 1 & 50 & 0.300 & 0.403 & 0.175 & 0.259 & 0.258 & 0.192 & 28.289 \\
2 & 50 & 0.300 & & 0.392 & 0.175 & 0.175 & 0.362 & 31.961 \\
3 & 20 & 0.750 & & & 0.552 & 0.258 & 0.222 & 16.368 \\
4 & 18 & 0.833 & & & & 0.562 & 0.224 & 15.788 \\
\hline
\end{tabular}

${ }^{1}$ Multiplied by (-100).

interval was assumed to be 5 yr to reflect a situation that can be found in dairy cattle breeding; hence, the number of generations was $T=10$. All possible 2-breed combinations to form a synthetic breed were analyzed with respect to the expected future diversity. To find the optimum contributions to the synthetic breed, the contributions varied in increments of 0.1 .

The real data set consisted of 13 central European red and yellow cattle breeds (Table 2). On average, 64 individuals per breed were sampled and were genotyped for 26 microsatellite markers. The data set is described by Simianer (2005b) and in detail by Bremond et al. (2002). Estimating real extinction probabilities is a difficult task because of the substantial amount of data required on the trend of population sizes over time (Bennewitz and Meuwissen, 2005b). Here, such detailed data were not available and it is assumed that the number of female breeding animals $\left(N_{f}\right)$ is the main factor affecting the probability of extinction, and the assigned probabilities were

$$
z=\gamma_{f} \times \frac{1}{N_{f}},
$$

with $\gamma_{f}=35$. Similar to the choice of $\gamma$ above, the factor $\gamma_{f}$ was chosen to let the extinction probabilities vary within a reasonable range. It resulted in probabilities between 0.875 for the most endangered breed (Thueringer Rotvieh) and $<0.001$ for the largest breed (Angler Rotvieh modern type); see Table 2 . It was assumed that they were valid for $t=50 \mathrm{yr}$. The number of breeding females and the effective population size were taken from Simianer (2005b). The generation interval was assumed to be $5 \mathrm{yr}$. The genotypes of the markers were used to estimate a kinship matrix using a weighted loglinear model (Eding and Meuwissen, 2003). The relative contributions to the MVT core set and the marginal diversities were estimated as described above, but improved contributions were obtained by bootstrapping as described by Bennewitz and Meuwissen (2005a).

It is assumed that conservation of breeds as a closed population is not desired for breeds with an extinction probability above 0.8 ; hence, no conservation effort will be spent on Czech Red Cattle and Thueringer Rotvieh (Table 1). Therefore, in the described iterative procedure to find breed combinations for synthetic breeds at least one of these breeds was included $(m=2)$. Optimal

Table 2. Effective population size $\left(N_{e}\right)$, number of registered breeding females, extinction probability $(z)$, relative breed contribution to the maximum-variance-total (MVT) core set $\left(c_{m v t}\right)$, and marginal diversity $(m d)$ of the breeds from the real data set

\begin{tabular}{lrrrrr}
\hline Breed & \multicolumn{1}{c}{$N_{e}{ }^{1}$} & \multicolumn{1}{c}{$N_{f}^{1}$} & \multicolumn{1}{c}{$z$} & $c_{m v t}$ & $m d^{2}$ \\
\hline Angler Rotvieh old type & 31.3 & 344 & 0.101 & 0.032 & 1.029 \\
Angler Rotvieh modern type & 237.8 & 15,300 & $<0.001$ & 0.144 & 0.420 \\
West German Angler Rotvieh & 78.3 & 900 & 0.039 & 0.009 & 0.142 \\
Gelbvieh & 180.8 & 7,500 & $<0.001$ & 0.097 & 0.226 \\
Glanvieh & 184.6 & 600 & 0.058 & 0.027 & 0.078 \\
Harzer Rotvieh & 26.5 & 125 & 0.280 & 0.022 & 1.053 \\
Thueringer Rotvieh & 23.8 & 40 & 0.875 & 0.029 & 1.250 \\
Limpurger & 68.4 & 118 & 0.297 & 0.223 & 1.157 \\
Podgorska Red Cattle & 75.0 & 300 & 0.117 & 0.004 & 0.054 \\
Rotes Hoehenvieh & 38.3 & 220 & 0.159 & 0.119 & 0.746 \\
Czech Red Cattle & 14.6 & 42 & 0.833 & 0.272 & 3.857 \\
Vogtlaender Rotvieh & 24.8 & 55 & 0.636 & 0.012 & 0.823 \\
Wittgensteiner Rotvieh & 26.7 & 140 & 0.250 & 0.008 & 1.129 \\
\hline
\end{tabular}

${ }^{1}$ Taken from Simianer (2005a).

${ }^{2}$ Multiplied by (-100). 
Table 3. Optimal breed contributions to a synthetic breed $\left(c_{s}\right)$, expected future maximum-variance-total (MVT) diversity $\left[E\left(D_{t}\right)\right]$, expected future within-breed diversity $\left[E\left(D_{w, t}\right)\right]$ and expected future between-breed diversity $\left[E\left(D_{b, t}\right)\right]$ when combining breeds with optimal contributions

\begin{tabular}{lcccc}
\hline $\begin{array}{l}\text { Breed } \\
\text { combination }\end{array}$ & $c_{s}$ & $E\left(D_{t}\right)$ & $E\left(D_{w, t}\right)$ & $E\left(D_{b, t}\right)$ \\
\hline 1 and 2 & $0.4,0.6$ & 0.643 & 0.553 & 0.090 \\
1 and 3 & $0.7,0.3$ & 0.702 & 0.531 & 0.171 \\
1 and 4 & $0.7,0.3$ & 0.711 & 0.526 & 0.185 \\
2 and 3 & $0.8,0.2$ & 0.698 & 0.541 & 0.157 \\
2 and 4 & $0.9,0.1$ & 0.707 & 0.524 & 0.183 \\
3 and 4 & $0.5,0.5$ & 0.741 & 0.519 & 0.222 \\
\hline
\end{tabular}

contributions to the synthetic breed were found as described above. The extinction probability of synthetic breeds were determined as

$$
z_{s}=\gamma_{f} \times \frac{1}{\sum_{i=1}^{m} N_{f, i}} .
$$

\section{RESULTS}

The relative breed contributions to the core set, marginal diversities, and the kinships of the simulated data set are shown in Table 1. All 4 breeds contributed significantly to the actual diversity. The greatest contribution and the greatest marginal diversity were obtained by breed 2 . The contributions of breeds 3 and 4 were very similar because of their similar kinships. The actual diversity was $D=0.923$, with $D_{w}=0.532$ and $D_{b}=$ 0.391 . The corresponding figures for the expected future diversity were $0.703,0.482$, and 0.221 , respectively. Hence, the loss of MVT diversity over time was around $24 \%$, which was much greater for the between-breed diversity (43\%) than for the within-breed diversity (9.5\%). The expected future diversity when forming a synthetic breed by combining 2 breeds are shown in Table 3 for every 2-breed combination with optimal contributions. The combination of breeds 1 and 4, 2 and 4 , and especially 3 and 4 resulted in an increase of expected future diversity, which is always because of an increase in the expected within-breed diversity. The optimal contributions to the synthetic breed tended to be larger for those breeds that were more important for the conservation of diversity; that is, showed a greater marginal diversity (Tables 1 and 3). On the contrary, the combination of the less endangered breeds 1 and 2 resulted in a loss of diversity, especially of the betweenbreed diversity (Table 3).

The breed contributions to the MVT core set and the marginal diversities of the red and yellow breeds are shown in Table 2. Every breed contributed to the actual diversity, highest contributions were obtained by the Czech Red and the Limpurger. The Czech Red, which was one of the most endangered breeds, also showed the largest marginal diversity. The MVT diversity was $D=1.028$, with $D_{w}=0.886$ and $D_{b}=0.142$. Here, the corresponding figures for the expected future diversity were $1.081,0.784$, and 0.297 , respectively. Hence, the MVT diversity increased over time by around $5 \%$, which is due to the increase of the between-breed diversity by inbreeding. The results of forming a synthetic breed with the Czech Red are shown in Table 4. The combination of Czech Red and West German Angler with contributions of 1 and 0 , respectively, increased $E\left(D_{t}\right)$ by $0.21 \%$. Other contributions resulted in a lower $E\left(D_{t}\right)$. Also, a putative combination of Czech Red and Thueringer Rotvieh, Podgorska Red, or Vogtlaender Rotvieh resulted in a slight increase of $E\left(D_{t}\right)$ (Table 4). No putative partner was found for the second most endangered breed, Thueringer Rotvieh (not shown), except Czech Red (Table 4). Also, after the combination of the Czech Red and the West German Angler, no further improvement of diversity was obtained.

\section{DISCUSSION}

First, the limits of the results from the field data set have to be acknowledged. From equations [1] and [6] it follows that the estimation of $E\left(D_{t}\right)$ depends on the extinction probabilities $(z)$ and the effective population size $\left(N_{e}\right)$ of the breeds. For the real data set, it was assumed that $z$ depends solely on the present size of female breeding population (equations [8a] and [8b]), which is certainly an oversimplification, because it ignores the historical and recent trend in the population size (Bennewitz and Meuwissen, 2005b). However, such data were not available here. Further, the determination of $z$ of a synthetic breed (equation [8b]) implies that all farmers keeping one of the $m$ breeds are switching to the synthetic breed in the long-term, again ignoring the trend in the size of the $m$ breeds. It remains to be investigated how the $z$ of the synthetic breed behaves under different breed contributions with different (trends in) population sizes. The figures for $N_{e}$ in Table 2 depended on the number of breeding males and females (Simianer, 2005b), which is also a simplification. Hence, because of these inaccuracies in the $z$ and $N_{e}$ figures, the numerical results of the field data set should be regarded solely as a demonstration of the methods.

From the simulation results (Table 3), it follows that a combination of breeds resulted, in general, in a loss of $E\left(D_{b, t}\right)$ and an increase in $\mathrm{E}\left(D_{w, t}\right)$. A combination of breeds seems to be advisable only if at least one breed is severely endangered. Only in these cases the loss of $\mathrm{E}\left(D_{t}\right)$ due to extinction of breeds would be greater than 
Table 4. Results of a combination of the Czech Red with one other breed showing marker-estimated kinship between the partner and the Czech Red $(f)$, optimal breed contributions to the synthetic breed $\left(c_{s}\right)$, expected future maximum-variance-total (MVT) diversity $\left[E\left(D_{t}\right)\right]$, expected future within-breed diversity $\left[E\left(D_{w, t}\right)\right]$, and expected future between-breed diversity $\left[E\left(D_{b, t}\right)\right]$ when combining breeds with optimal contributions

\begin{tabular}{lccccc}
\hline Czech Red combined with & $f$ & $c_{s}{ }^{1}$ & $E\left(D_{t}\right)$ & $E\left(D_{w, t}\right)$ & $E\left(D_{b, t}\right)$ \\
\hline Angler Rotvieh old type & 0.036 & $0.9,0.1$ & 1.076 & 0.798 & 0.278 \\
Angler Rotvieh modern type & 0.034 & $1.0,0.0$ & 1.080 & 0.795 & 0.285 \\
West German Angler & 0.060 & $1.0,0.0$ & 1.083 & 0.792 & 0.291 \\
Gelbvieh & 0.011 & $1.0,0.0$ & 1.079 & 0.794 & 0.285 \\
Glanvieh & 0.030 & $1.0,0.0$ & 1.079 & 0.794 & 0.285 \\
Harzer Rotvieh & 0.031 & $0.9,0.1$ & 1.079 & 0.793 & 0.286 \\
Thueringer Rotvieh & 0.034 & $0.8,0.2$ & 1.082 & 0.786 & 0.296 \\
Limpurger & 0.009 & $0.9,0.1$ & 1.075 & 0.793 & 0.282 \\
Podgorska Red Cattle & 0.059 & $1.0,0.0$ & 1.082 & 0.792 & 0.290 \\
Rotes Hoehenvieh & 0.034 & $1.0,0.0$ & 1.081 & 0.791 & 0.290 \\
Vogtlaender Rotvieh & 0.051 & $1.0,0.0$ & 1.082 & 0.788 & 0.294 \\
Wittgensteiner Rotvieh & 0.039 & $1.0,0.0$ & 1.081 & 0.791 & 0.290 \\
\hline
\end{tabular}

${ }^{1}$ The first value denotes the contribution of the Czech Red.

the general loss of $E\left(D_{b, t}\right)$ when forming the synthetic breed. For example, the combination of breeds 1 and 2 , both with $z=0.3$, resulted in a substantial loss of $E\left(D_{t}\right)$ because of the loss of $E\left(D_{b . t}\right)$, but the combinations with at least 1 of the 2 endangered breeds 3 and 4 resulted, in most cases, in a greater $E\left(D_{t}\right)$. Note that the increase of $E\left(D_{b, t}\right)$ when combining breeds 3 and 4 was an exception in this study and was due to the high extinction probability of both breeds (Table 1). Suitable partners for endangered breeds should ideally be closely related because this results in a reduced loss of $E\left(D_{b, t}\right)$. In the simulated data set these are either breed combinations 3 and 4 or one of them with breed 1 , which are all related (Table 1), and all combinations increased $E\left(D_{t}\right)$ (Table 3). On the contrary, a combination of breed 3 with breed 2 (both are not related, Table 1 ) would result in a loss of $E\left(D_{t}\right)$ of $30 \%$ (Table 3 ) and would not be beneficial. The results of the real data confirm this. The highest between-breed kinships of Czech Red were obtained by the West German Angler (Table 4), and hence, this breed was the best partner for the Czech Red. The other putative partners of the Czech Red (Thueringer Rotvieh, Podgorska Red, and Vogtlaender Rotvieh), which resulted in an increase in $E\left(D_{t}\right)$, were also related to it (Table 4). A combination of the Czech Red and the Limpurger resulted in a loss of diversity, because both breeds are not related and, hence, much of the between-breed diversity was lost (Table 4).

If 2 putative breed partners show a similar high kinship with the endangered breed, but one itself is endangered and the other is not, the more endangered breed is to be preferred, because this combination increases diversity contributed by both partners. A combination with the less endangered breed partner hardly affects the $z$ of this partner; hence, its contribution to future diversity is reduced because of the loss of $E\left(D_{b, t}\right)$. This can be shown when comparing the $E\left(D_{t}\right)$ of breed combi- nations 1 and 4 and 3 and 4, respectively (Table 3). The kinships between both breeds in the combinations were the same (Table 1) but because of the higher $z$ of breed 3 compared with breed 1 , the combination of 3 and 4 was superior.

Of course, an endangered breed has to be relevant for the conservation of diversity. For example, although it might be advisable to form a synthetic breed with the Czech Red included, no combination with the second highly endangered breed, the Thueringer Rotvieh, resulted in an increase in $E\left(D_{t}\right)$ (except the combination of both). The reason is that the Czech Red is important for the conservation of diversity whereas the Thueringer Rotvieh is not, as can be seen from the relative breed contributions to the MVT core set and from the marginal diversities (Table 2).

The question of what proportion a breed should contribute to the synthetic breed was answered by choosing proportions that maximized $E\left(D_{t}\right)$, which led to contributions that were roughly proportional to the ratio of the marginal diversities of the breeds (Table 1). When determining optimal contributions for breeds with similar marginal diversities, it was, in general, observed that with extreme contributions $\left[\mathbf{c}_{\mathbf{r}}^{\prime}=(1,0)\right.$ or $\mathbf{c}_{\mathbf{r}}^{\prime}=(0$, $1)$ ], the $E\left(D_{b, t}\right)$ reached its maximum, whereas with intermediate contributions $\left[\mathbf{c}_{\mathbf{r}}^{\prime}=(0.5,0.5)\right]$, the $E\left(D_{w, t}\right)$ was maximized (see Figure 1 for an example from the simulation). Following this, there is a trade-off between these components when finding optimal contributions. However, the differences were only small, which offers the opportunity to choose those contributions stakeholders can agree upon (e.g., proportionally to the population size) without losing too much diversity, providing that both breeds show similar marginal diversities.

The extreme optimal contributions of the Czech Red and one of the other breeds (Table 4) are due to the very high marginal diversity of the Czech breed (Table 


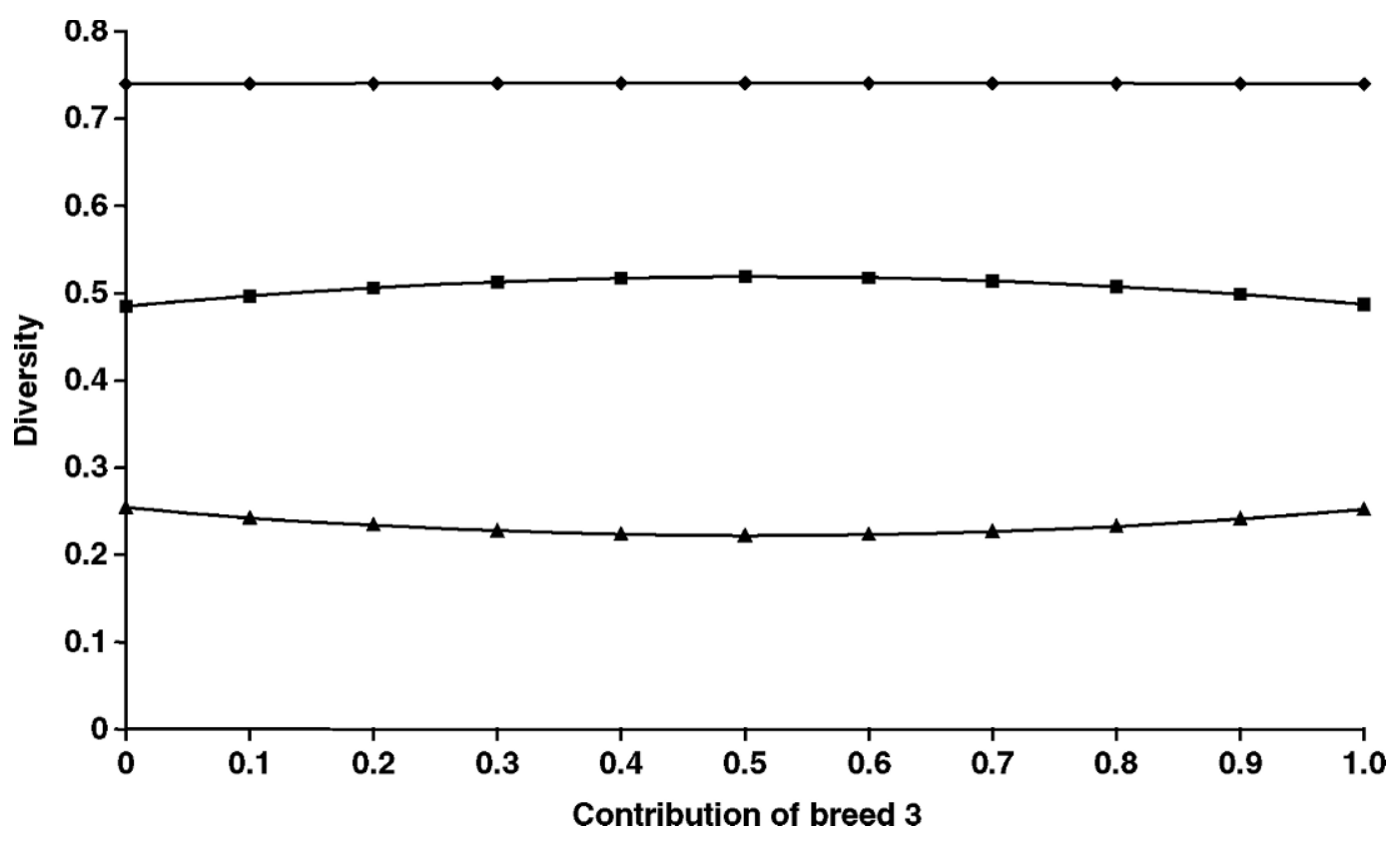

Figure 1. Expected future maximum-variance-total diversity ( $\bullet$, top line), expected future within-breed diversity ( $\mathbf{\square}$, middle line), and expected future between-breed diversity ( $\boldsymbol{\Lambda}$, bottom line) when combining breeds 3 and 4 from the simulated data set with different contributions. The contribution of breed 4 is 1 minus the contribution of breed 3 .

2). A contribution of $\mathbf{c}_{\mathbf{r}}^{\prime}=(1,0)$, as for the Czech Red and the West German Angler (Table 4), suggests not a combination of these 2 breeds, but a complete replacement of the West German Angler by the Czech Red. The small increase of $E\left(D_{t}\right)$ for this particular situation is because of the reduced extinction probability of the Czech Red (from 0.833 to 0.037, see Table 2 and equation [8b]) if it replaces the West German Angler completely. Putative contributions to a synthetic breed formed by the Thueringer Rotvieh and one of the other breeds were in general much less extreme (not shown), which is due to the intermediate marginal diversity of the Thueringer Rotvieh (Table 2).

It should be noted that the suggested complete replacement of the West German Angler led to an increase of the expected future diversity $\left[E\left(D_{t}\right)\right]$, but an elimination of this breed did not increase the actual diversity $\left(D_{0}\right)$. The reason is that $D_{0}$ does not consider extinction probabilities (in contrast to $E\left(D_{t}\right)$, see equations [1] to [4]), and, because of the nature of the MVT core set, it never increases if a breed is removed (Bennewitz and Meuwissen, 2005a).

The increase of MVT diversity over time in the field data set might be surprising and intuitively a decline due to extinction events would be expected. However, from equation [6a], it follows that with many nonendangered breeds included in the set, genetic drift might cause an increase of $D_{b}$ over time. In this study the increase in $D_{b}$ was around $100 \%$ compared with time $t=0$ and overcompensated the loss of diversity caused by extinction and the loss of $D_{w}$ due to drift. Note that putative inbreeding depressions, which might lower the diversity, are not accounted for in estimating the expected future diversity.

As mentioned previously, the MVT diversity is defined as the genetic variance of a quantitative trait that can be found within and between breeds and, thus, is based on a quantitative genetic argument (Bennewitz and Meuwissen, 2005a). The weights given to the within- and between-breed variance are equal (equations [6a] and [6b]). Other diversity measures differ from the MVT diversity mainly in the weighting of betweenbreed and within-breed diversity. The Weitzman diversity measure (Weitzman, 1992) considers solely the between-breed diversity and ignores the within-breed diversity. However, within-breed diversity can be substantial in livestock breeds, as shown by the results from the field data set. Because overall a combination of breeds resulted in a loss of between-breed diversity (Table 3), the use of this diversity measure would suggest not combining any breeds. On the contrary, the measure of Eding et al. (2002) defines diversity as the variance of a trait that can be found in putative offspring that are obtained from interbreeding conserved breeds. This measure would not be able to distinguish between a set of $r$ breeds versus a set of $r-m+1$ breeds, where $m$ of the breeds have been merged, because it assumes that eventually all breeds are interbred. It 
may be noted that the Eding diversity measure is equivalent to the expected heterozygosity in a hypothetical meta-population criterion, and compared with this, the MVT measure gives twice as much weight to betweenbreed diversity (Toro et al., 2006). The MVT diversity measure is in between these 2 extreme measures (Weitzman diversity and Eding diversity), implying that the results in this study are more general than they would have been if obtained by use of the 2 extreme diversity measures. This was confirmed by the results of Toro et al. (2006), who found that breed contributions were more equal for the MVT criterion than for any other criteria.

An objective of a conservation scheme might include not only genetic diversity but also more breed-specific arguments such as, for example, the cultural value of a breed or its adaptation to a specific production environment. The term "utility" has been used in this context by Simianer et al. (2003). Following them, the utility of a set of breeds is the weighted combination of conserved diversity and the additional arguments. The suggested approach for combining breeds can be expanded straightforwardly to these more elaborate objectives by estimating expected utilities using the equations of Simianer et al. (2003) instead of expected diversities.

\section{CONCLUSIONS}

As expected, it is generally advisable to keep endangered breeds in closed populations in genetic conservation schemes. However, in some cases, it might be very difficult or impossible to conserve a highly endangered breed in a closed population. If this breed is important for the diversity (i.e., it shows a high marginal diversity), it might be beneficial to merge it with one or more (endangered) breeds to conserve a part of the diversity that is contributed by the highly endangered breed. The potential breed partner(s) should be closely related to the breed to minimize the loss of between-breed diversity. The contribution of each breed to the synthetic breed should be in accordance with their marginal diversities. Provided that reliable estimates for the extinction probability and the effective population size are available, the suggested approach can be used to identify those breed combinations with optimal contributions that result in an increase of conserved diversity. The behavior of the extinction probability of a synthetic breed needs to be investigated in more detail.

\section{ACKNOWLEDGMENTS}

Jörn Bennewitz was supported by a grant from the Deutsche Forschungsgemeinschaft, DFG.

\section{REFERENCES}

Bennewitz, J., and T. H. E. Meuwissen. 2005a. A novel method for the estimation of the relative importance of breeds in order to conserve the total genetic variance. Genet. Sel. Evol. 37:315-337.

Bennewitz, J., and T. H. E. Meuwissen. 2005b. Estimation of extinction probabilities of five German cattle breeds by population viability analysis. J. Dairy Sci. 88:2949-2961.

Bremond, J., H. Simianer, C. Drögemöller, A. Spötter, O. Distl, and A. Feldman. 2002. Molekulargenetische Differenzierung verschiedener Rotviehpopulationen. Schriftenreihe des Bundesministeriums für Verbraucherschutz, Ernährung und Landwirtschaft, Reihe Angewandte Wissenschaft, Vol. 493. Landwirtschaftsverlag, Münster-Hiltrup, Germany.

Eding, H., and T. H. E. Meuwissen. 2001. Marker based estimates of between and within population kinships for the conservation of genetic diversity. J. Anim. Breed. Genet. 118:141-159.

Eding, H., and T. H. E. Meuwissen. 2003. Linear methods to estimate kinships from genetic marker data for the construction of core sets in genetic conservation schemes. J. Anim. Breed. Genet. 120:289-302.

Eding, H., R. P. Crooijmans, M. A. Groenen, and T. H. E. Meuwissen. 2002. Assessing the contribution of breeds to genetic diversity in conservation schemes. Genet. Sel. Evol. 34:613-633.

Falconer, D. S., and T. F. C. MacKay. 1996. Introduction to Quantitative Genetics. Longman, London, UK.

Meuwissen, T. H. E. 2007. Operation of conservation schemes. Pages 167-194 in Utilisation and Conservation of Farm Animal Genetic Resources. K. Oldenbroek, ed. Wageningen Academic Publishers, Wageningen, the Netherlands.

Oldenbroek, K., ed. 2007. Utilisation and Conservation of Farm Animal Genetic Resources. Wageningen Academic Publishers, Wageningen, the Netherlands.

Scherf, B., B. Rischkowsky, D. Pilling, and I. Hoffmann. 2006. The state of the world's animal genetic resources. Proc. 8th World Congr. Genet. Appl. Livest. Prod., CD-ROM Commun. no. 33-13.

Simianer, H. 2005a. Decision making in livestock conservation. Ecol. Econ. 54:559-572.

Simianer, H. 2005b. Using expected allele number as objective function to design between and within breed conservation of farm animal biodiversity. J. Anim. Breed. Genet. 122:177-187.

Simianer, H., S. B. Reist-Marti, J. Gibson, O. Hanotte, and J. E. O. Rege. 2003. An approach to the optimal allocation of conservation funds to minimize loss of genetic diversity between livestock breeds. Ecol. Econ. 45:377-392.

Toro, M. A., J. Fernández, and A. Caballero. 2006. Scientific policies in conservation of farm animal genetic resources. Proc. 8th World Congr. Genet. Appl. Livest. Prod., CD-ROM Commun. no. 33-05.

Weitzman, M. L. 1992. On diversity. Q. J. Econ. 107:363-405.

Weitzman, M. L. 1993. What to preserve? An application of diversity theory to crane conservation. Q. J. Econ. CVII:157-183.

Woolliams, J., and M. Toro. 2007. What is genetic diversity? Pages 55-74 in Utilisation and Conservation of Farm Animal Genetic Resources. K. Oldenbroek, ed. Wageningen Academic Publishers, Wageningen, the Netherlands. 